\title{
Artrosis rápidamente progresiva de cadera secundaria a una fractura por insuficiencia subcondral del acetábulo. Reporte de un caso
}

\author{
Carlos M. Lucero, Fernando Díaz Dilernia, Gerardo Zanotti, Fernando Comba, \\ Francisco Piccaluga, Martín Buttaro \\ Centro de Cadera "Sir John Charnley", Instituto de Ortopedia y Traumatología "Prof. Dr. Carlos E. Ottolenghi”, \\ Hospital Italiano de Buenos Aires, Ciudad Autónoma de Buenos Aires, Argentina
}

\begin{abstract}
RESUMEN
Las fracturas por insuficiencia subcondral son una causa poco frecuente de cadera dolorosa. A diferencia de las fracturas traumáticas agudas, las fracturas por insuficiencia del acetábulo son menos frecuentes que las femorales. Ocurren habitualmente en mujeres posmenopáusicas con comorbilidades. Su diagnóstico inicial suele ser dificultoso y la sospecha clínica es de gran importancia. La resonancia magnética es una herramienta fundamental para detectar este cuadro. Subestimar estas lesiones puede llevar al desarrollo de una artrosis rápidamente progresiva y al reemplazo articular como desenlace. Se presenta el caso de una paciente de 68 años con una fractura por insuficiencia subcondral del acetábulo a quien se le indicó una artroplastia total de cadera no cementada.
\end{abstract}

Palabras clave: Fractura por insuficiencia; acetábulo; artroplastia total de cadera.

Nivel de Evidencia: IV

Rapidly Progressive Osteoarthritis of the Hip Secondary to a Subchondral Insufficiency Fracture of the Acetabulum: A Case Report

\section{ABSTRACT}

Subchondral insufficiency fractures are a rare cause of hip pain. Unlike acute traumatic fractures, acetabulum insufficiency fractures are less common than femoral fractures. They commonly occur in postmenopausal women with comorbidities. Its initial diagnosis is usually difficult and clinical suspicion is of great importance. Magnetic resonance imaging (MRI) is a fundamental tool for the detection of this pathology. Underestimating these injuries can lead to the development of rapidly progressive osteoarthritis and joint replacement as an outcome. We present the case of a 68-year-old patient with a subchondral insufficiency fracture of the acetabulum who underwent uncemented total hip arthroplasty.

Key words: Insufficiency fracture; acetabulum; total hip arthroplasty.

Level of Evidence: IV

\section{INTRODUCCIÓN}

Las fracturas subcondrales por insuficiencia son una causa infrecuente de coxalgia en el adulto. Hay diversos reportes publicados sobre fracturas subcondrales de la cabeza femoral, pero se ha descrito poco sobre la enfermedad por insuficiencia en el acetábulo. ${ }^{1,2}$

Habitualmente se producen en mujeres posmenopáusicas con comorbilidades y suelen afectar la columna, la pelvis y los miembros inferiores. Este patrón de fractura suele ocurrir de manera atraumática y, aunque las causas que la producen no están del todo definidas, se han comunicado diversos factores, como la corticoterapia prolongada, la enfermedad renal, la obesidad y las alteraciones del balance lumbopélvico. ${ }^{3}$

Recibido el 5-4-2020. Aceptado luego de la evaluación el 21-5-2020 • Dr. CARLOS M. LUCERO • lucerooyt@ gmail.com (ID https://orcid.org/0000-0002-0833-7818 Cómo citar este artículo: Lucero CM, Díaz Dilernia F, Zanotti G, Comba F, Piccaluga F, Buttaro M. Artrosis rápidamente progresiva de cadera secundaria a una fractura por insuficiencia subcondral del acetábulo. Reporte de un caso. Rev Asoc Argent Ortop Traumatol 2021;86(2):228-234. https://doi.org/10.15417/issn. 1852-7434.2021.86.2.1090 
Este cuadro suele desestimarse, debido a que, en los estadios iniciales, la ausencia de signos radiográficos claros dificulta el diagnóstico precoz. La sospecha de esta lesión es fundamental e identificarla, de forma temprana, puede evitar una artrosis rápidamente progresiva, cuyo desenlace será el reemplazo articular. ${ }^{4}$

Presentamos el caso de una mujer de 68 años con artrosis rápidamente progresiva de cadera secundaria a una fractura por insuficiencia del acetábulo sin traumatismo asociado, tratada con una artroplastia total de cadera no cementada.

\section{CASO CLÍNICO}

Una mujer de 68 años de edad consultó en nuestro centro por coxalgia derecha de un año de evolución, sin antecedente traumático. Su índice de masa corporal era de 30,9 y no refería consumo crónico de medicación, alcohol o tabaco, ni otras comorbilidades de relevancia, así como tampoco cirugías ortopédicas previas. Antes del comienzo de los síntomas, la paciente se valía por sus propios medios, con un nivel 6/6 en las actividades de la vida diaria. Cuando se la evaluó, tenía un índice de Katz 4/6. El dolor, principalmente mecánico, se había incrementado en los últimos tres meses, lo que la obligó a usar un bastón y a recurrir al consumo de analgésicos. En el examen físico de la cadera derecha, la flexión máxima era de $145^{\circ}$; la extensión, de $20^{\circ}$; la rotación interna, de $40^{\circ}$; la rotación externa, de $50^{\circ}$; la abducción, de $40^{\circ}$ y la aducción, de $25^{\circ}$. No había discrepancia aparente ni desviaciones en el eje mecánico de ambos miembros inferiores.

En las radiografías anteroposteriores y de perfil de ambas caderas, se observó un ángulo cervicodiafisario de $131^{\circ}$, con un índice acetabular y un ángulo centro de borde lateral de Wiberg dentro de los límites normales en ambas caderas. En la cadera derecha, se observaron signos evidentes de desgaste articular con un estrechamiento de la luz articular y esclerosis subcondral, clasificada como grado 2 de Tönnis. Observando con mayor detenimiento, se detectó un escalón articular acetabular a nivel posterosuperior (Figura 1). Se solicitó una resonancia magnética (RM) cuyas imágenes revelaron una zona de hiperintensidad en el área de carga del acetábulo, lo que confirmó la lesión (Figura 2). Los parámetros bioquímicos no mostraron alteraciones significativas.

Sobre la base de estos hallazgos, se diagnosticó una fractura por insuficiencia subcondral del acetábulo derecho. En primera instancia y luego de haberle explicado el pronóstico probable, se decidió realizar una infiltración bajo tomografía computarizada con ácido hialurónico y un corticoide de depósito, y se le indicó continuar con descarga de la extremidad afectada mediante un bastón, durante seis semanas. El dolor mejoró levemente luego de tres meses, pero aún continuaba con importantes limitaciones para deambular más de 100 metros. Finalmente, luego de cuatro meses desde la consulta inicial en nuestro centro y ante la progresión de los síntomas, se sometió a una artroplastia total de cadera derecha no cementada (Figura 3). Los controles radiográficos a los tres y seis meses de la cirugía mostraron implantes estables y la paciente había reanudado su actividad habitual sin restricciones (Figura 4).

\section{DISCUSIÓN}

Las fracturas acetabulares, en general, se asocian con un traumatismo de alta energía. ${ }^{5}$ Por el contrario, una fractura por insuficiencia subcondral se produce por el estrés normal o fisiológico aplicado al tejido óseo con una resistencia elástica deficiente. 6

A diferencia de las fracturas traumáticas agudas, las fracturas por insuficiencia del acetábulo son extremadamente raras en comparación con las femorales. ${ }^{7}$ Se han publicado diversos factores predisponentes, entre ellos, el consumo crónico de corticoides, la osteopenia, el alcohol y un índice de masa corporal elevado; este último es el único hallazgo patológico en nuestro paciente. ${ }^{8,9}$

El diagnóstico a tiempo de estas lesiones es esencial, ya que, si no se diagnostican, pueden desencadenar una artrosis rápidamente progresiva y aumentar la morbimortalidad en estos pacientes..$^{10,11}$ Las radiografías de rutina, a menudo, no logran identificar estas fracturas, la osteopenia es el único hallazgo radiográfico evidenciable. Angles y cols. publicaron un caso de una fractura por insuficiencia del acetábulo en una paciente de 83 años, cuyas radiografías no mostraron alteraciones inicialmente y, luego de tres semanas de continuar con síntomas, se llegó al diagnóstico de la lesión acetabular mediante tomografía. ${ }^{12}$

Ante la consulta por coxalgia aguda sin antecedente traumático y sin anormalidades radiográficas visibles, se debe considerar la posibilidad de realizar una RM. Este estudio se caracteriza por su alta sensibilidad para detectar fracturas por insuficiencia acetabular y femoral, y brinda información adicional sobre otras patologías. Por ejemplo, el edema óseo sin una fractura podría corresponder a un edema transitorio de la médula ósea. En algunos casos, estos hallazgos en la RM podrían estar directamente relacionados con la evolución natural hacia una fractura por insuficiencia subcondral de la cadera. ${ }^{13}$ 

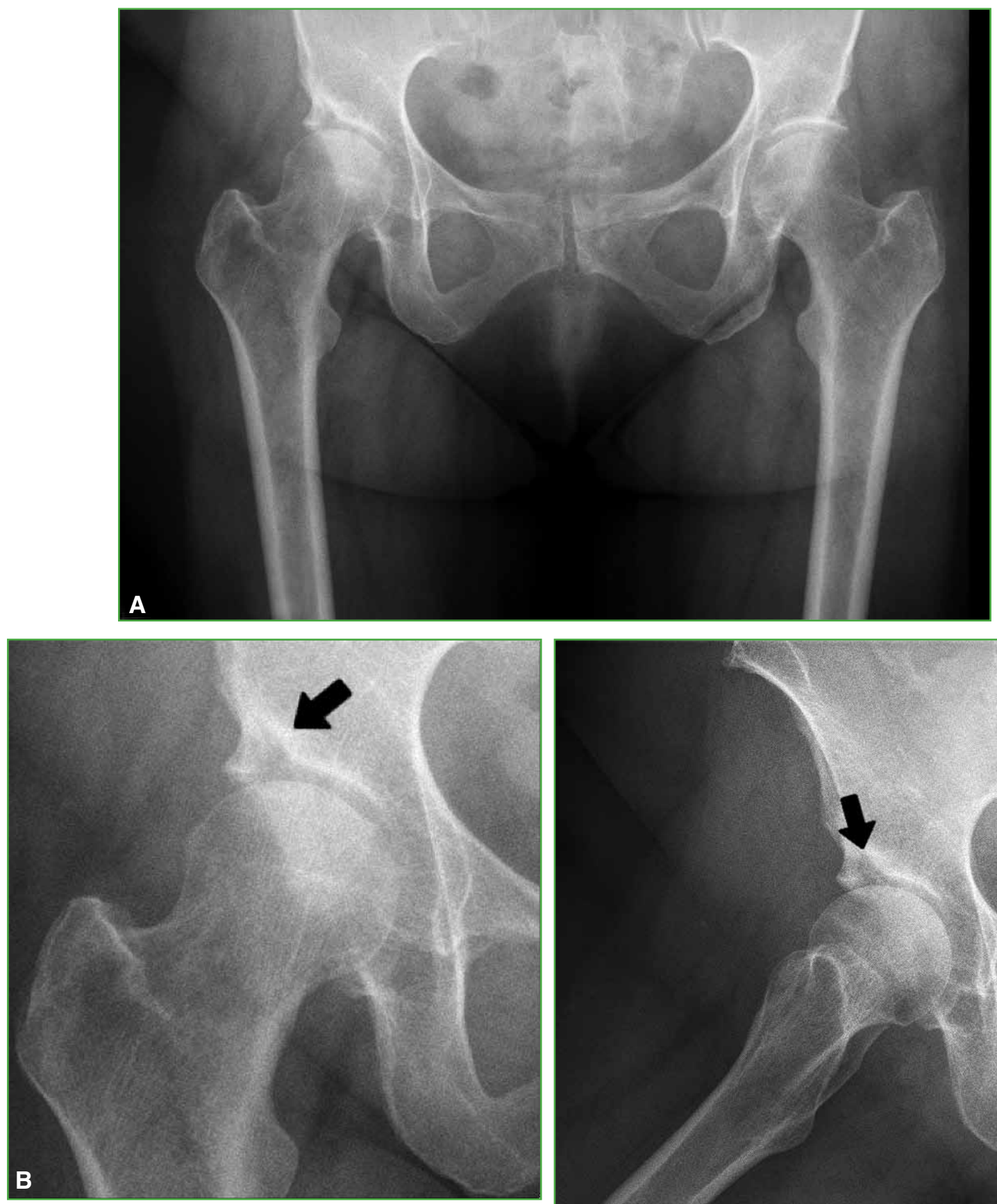

Figura 1. Radiografías de cadera derecha preoperatorias, anteroposterior (A), anteroposterior ampliada (B) y de perfil (C). Se observa el colapso subcondral focal de $2 \mathrm{~mm}$ de profundidad en la porción posterosuperior del techo acetabular (flechas).

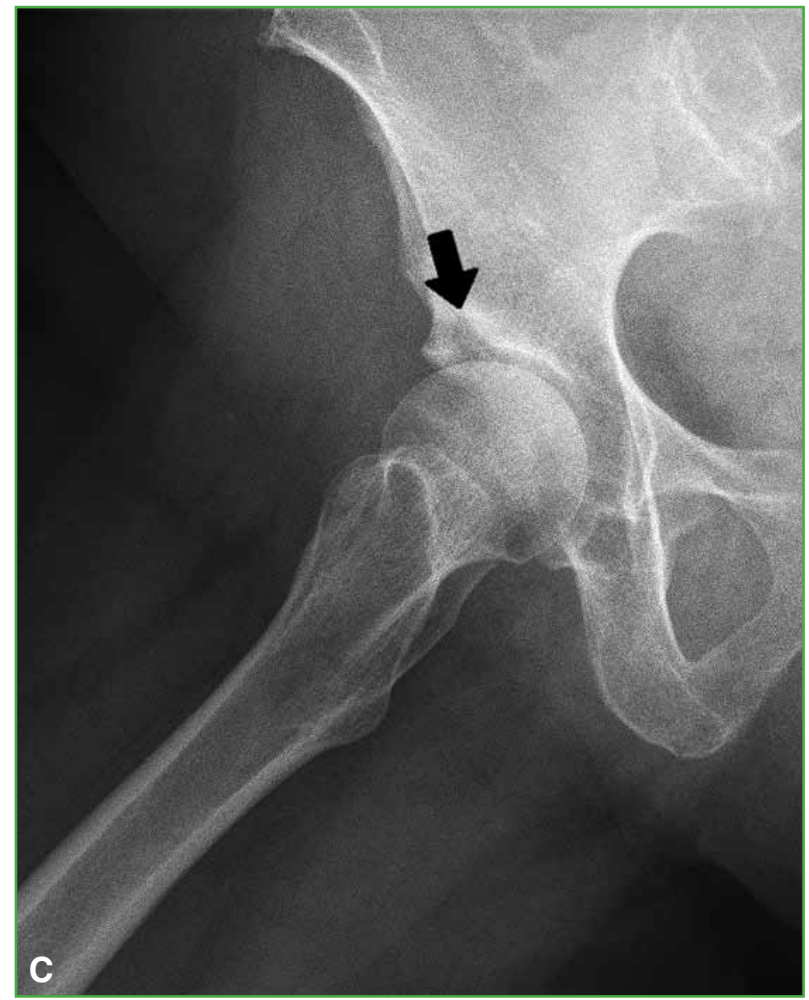



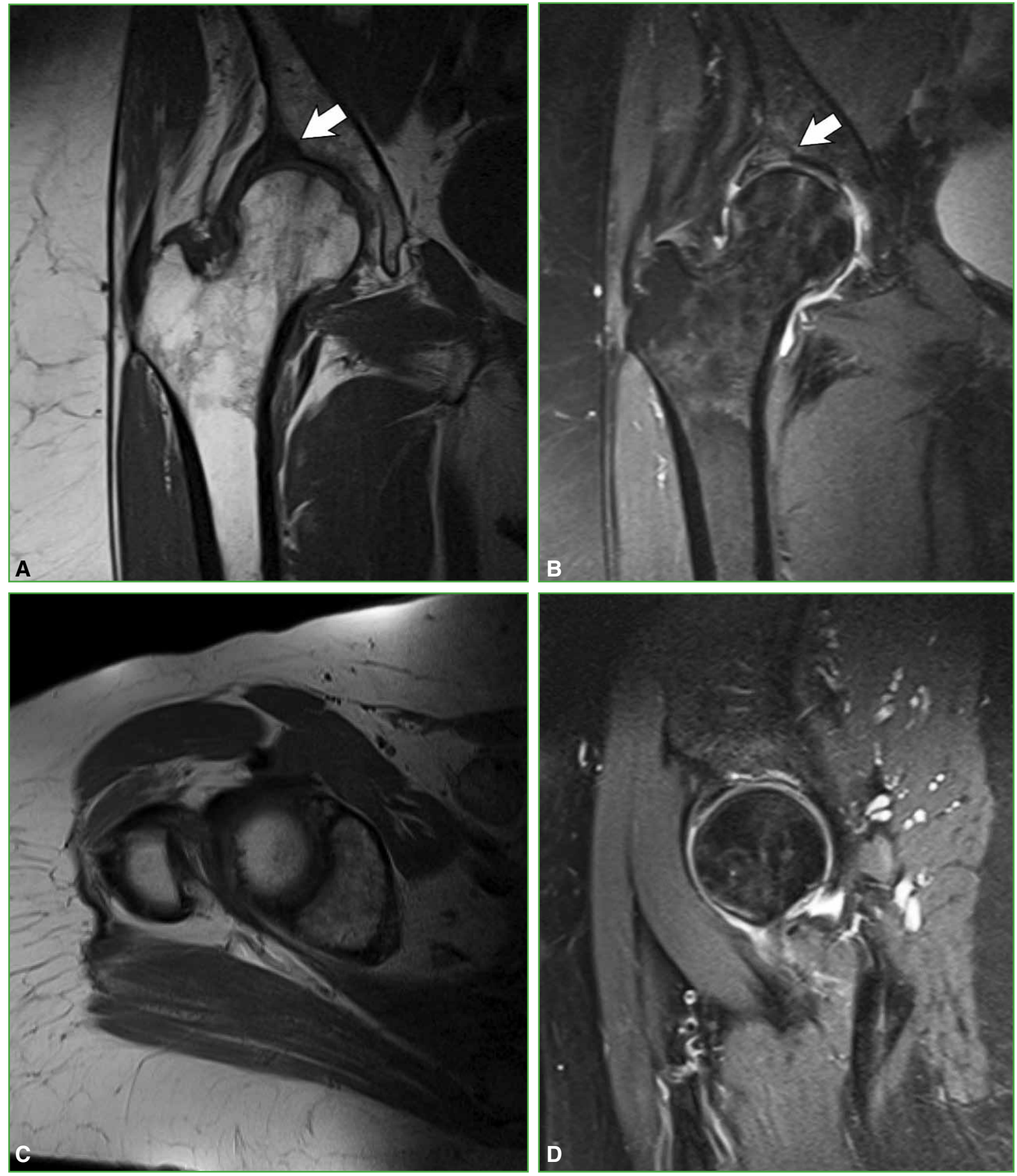

Figura 2. Resonancia magnética, cortes coronales (A y B), corte axial (C), corte sagital (D). Hipointensidad en secuencia T1 e hiperintensidad en secuencia T2. Edema periacetabular sugestivo de fractura por insuficiencia. 


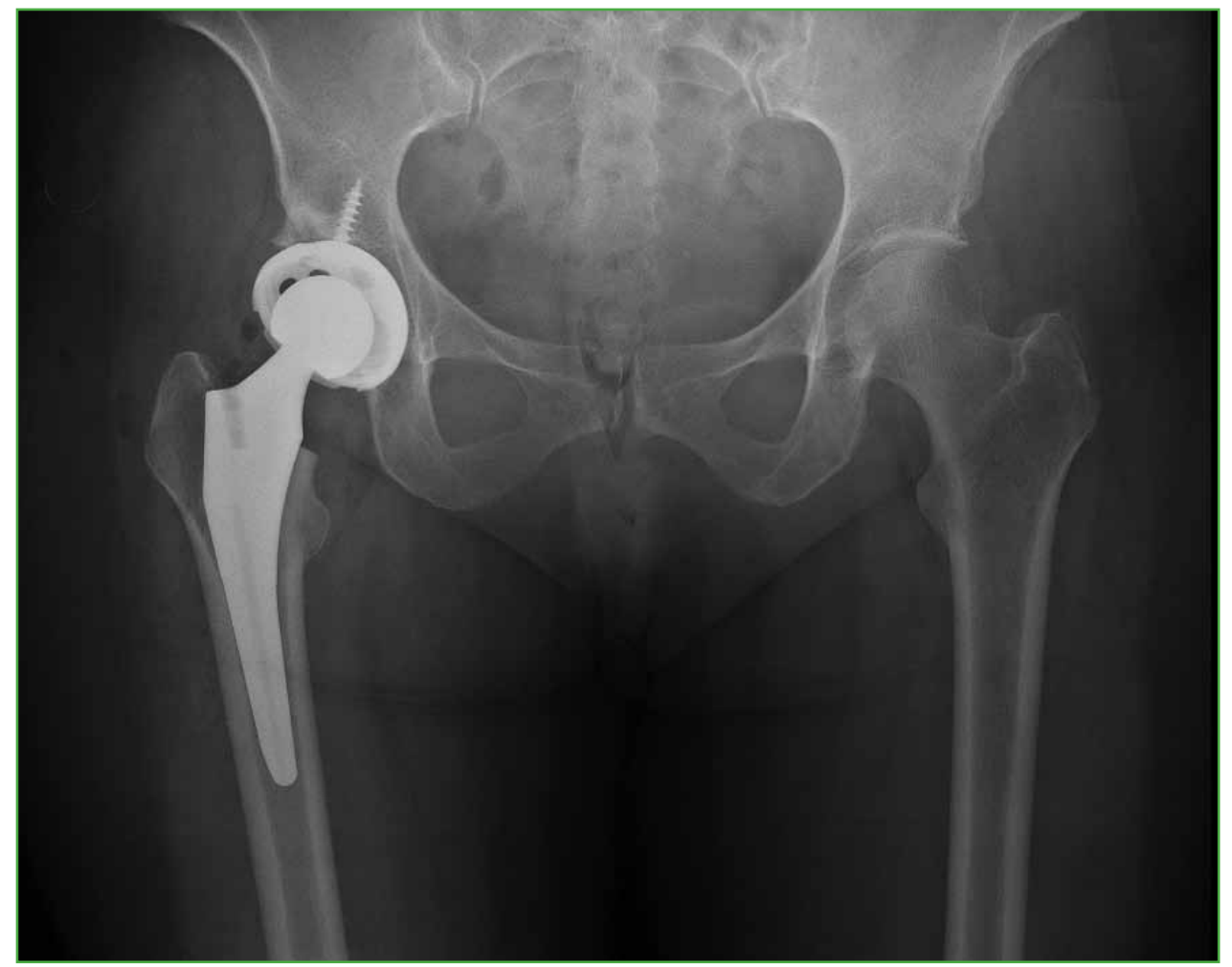

Figura 3. Radiografía posquirúrgica inmediata. Se observa el reemplazo total no cementado de la cadera derecha.

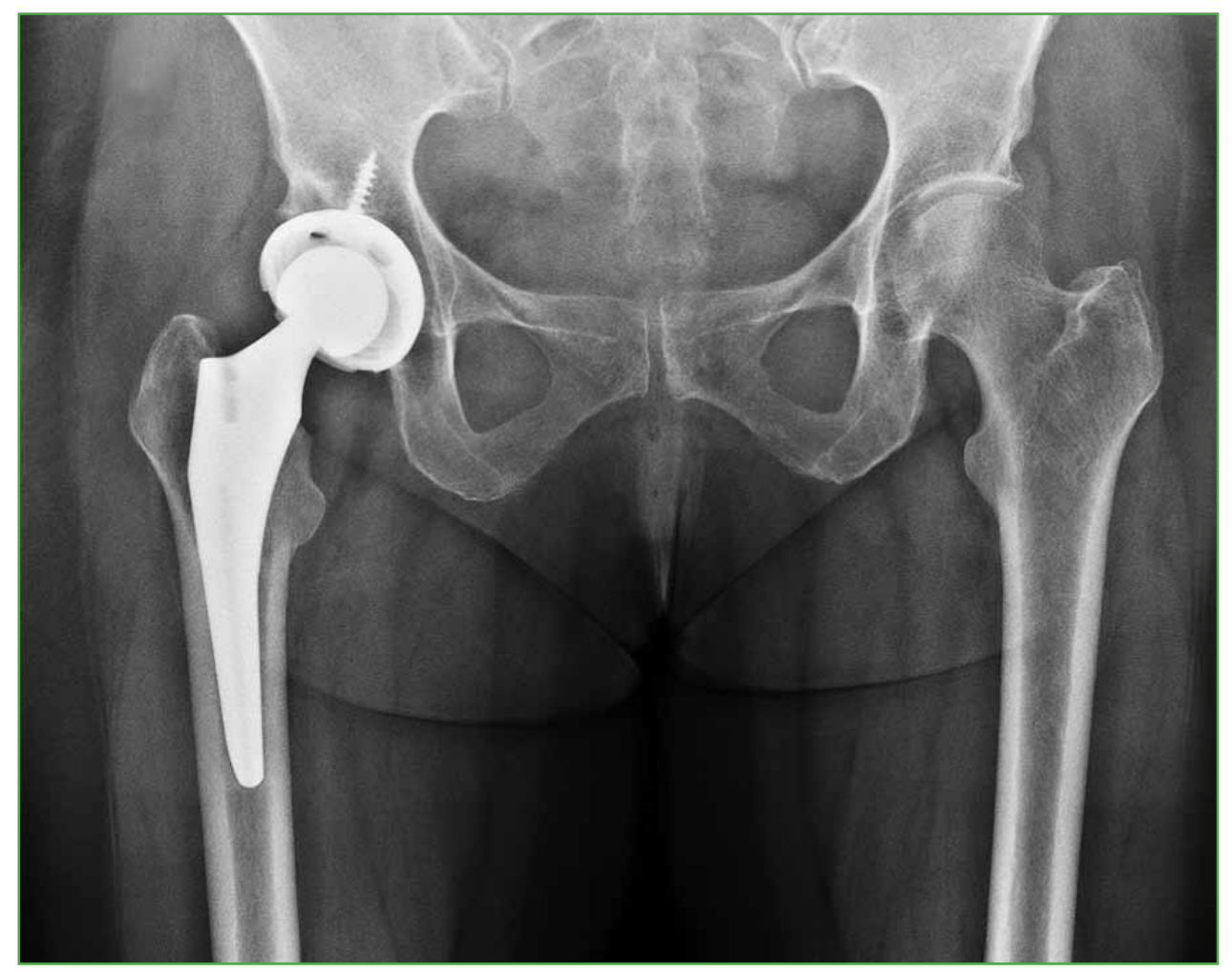

Figura 4. Radiografía a los 6 meses de la cirugía. 
Como diagnósticos diferenciales, se deben considerar las fracturas ocultas, el edema óseo, la osteoporosis transitoria, las lesiones osteocondrales y la osteonecrosis de cadera. ${ }^{14}$ Luego de la RM diagnóstica inicial, es necesario realizar un seguimiento seriado de estas lesiones, al menos, a las tres semanas del diagnóstico. ${ }^{15}$ Creemos que la tomografía debe reservarse para aquellos casos en los que hay un antecedente traumático o una cirugía homolateral previa. En este sentido, Guerado y cols. describieron una serie de pacientes $>75$ años a quienes se les había diagnosticado una fractura oculta del acetábulo secundaria a la osteosíntesis endomedular para tratar una fractura de cadera. ${ }^{16}$

No debe dejarse de lado la evaluación por imágenes de la columna, porque la cifosis lumbar degenerativa y otras alteraciones del balance espinopélvico podrían ser factores predisponentes. ${ }^{17} \mathrm{Sin}$ embargo, en el caso reportado, nuestra paciente no tenía alteraciones en el espinograma.

La evidencia conocida sobre el tratamiento de las fracturas acetabulares por insuficiencia es variada y las opciones terapéuticas dependen de la presentación clínica e incluyen métodos conservadores y quirúrgicos. ${ }^{18} \mathrm{El}$ manejo inicial de una fractura por insuficiencia del acetábulo o de la cabeza femoral es controvertido. Si no hay signos de colapso en los estudios por imágenes, algunos autores recomiendan la restricción de la carga de peso como primera línea de tratamiento. ${ }^{19}$ A nuestra paciente, luego del fracaso del tratamiento conservador, en primera instancia, se le indicó una artroplastia total de cadera no cementada.

Es importante la sospecha clínica ante un paciente con coxalgia aguda y factores predisponentes asociados, cuyas radiografías iniciales son normales. La RM sigue siendo una herramienta fundamental en el diagnóstico de estas lesiones, se debe solicitar de inmediato cuando se sospecha este cuadro para evitar el colapso articular y la artrosis rápidamente progresiva. ${ }^{20}$

Conflicto de intereses: Los autores no declaran conflictos de intereses.

ORCID de F. Díaz Dilernia: https://orcid.org/0000-0002-7830-2207 ORCID de G. Zanotti: https://orcid. org/0000-0001-8090-4832

ORCID de F. Piccaluga: https://orcid.org/0000-0002-9887-4886 ORCID de F. Comba: https://orcid. org/0000-0002-2848-2983

\section{BIBLIOGRAFÍA}

1. Kawano K, Motomura G, Ikemura S, Kubo Y, Hatanaka H, Utsunomiya T, et al. Subchondral insufficiency fracture of the femoral head in an elderly woman with symptomatic osteoarthritis of the contralateral hip. J Orthop Sci 2018;S0949-2658(18)30043-5. https://doi.org/10.1016/j.jos.2018.02.001

2. Kimura T, Goto T, Hamada D, Tsutsui T, Wada K, Fukuta S, et al. Subchondral insufficiency fracture of the femoral head caused by excessive lateralization of the acetabular rim. Case Rep Orthop 2016;2016:4371679. https://doi.org/10.1155/2016/4371679

3. Hackney LA, Lee MH, Joseph GB, Vail TP, Link TM. Subchondral insufficiency fractures of the femoral head: associated imaging findings and predictors of clinical progression. Eur Radiol 2016;26(6):1929-41. https://doi.org/10.1007/s00330-015-3967-x

4. Yamamoto T, Bullough PG. The role of subchondral insufficiency fracture in rapid destruction of the hip joint: a preliminary report. Arthritis Rheum 2000;43(11):2423-7. https://doi.org/10.1002/1529-0131(200011)43:11<2423::AID-ANR8>3.0.CO;2-Z

5. Jauregui JJ, Clayton A, Kapadia BH, Cherian JJ, Issa K, Mont MA. Total hip arthroplasty for acute acetabular fractures: a review of the literature. Expert Rev Med Devices 2015;12(3):287-95. https://doi.org/10.1586/17434440.2015.1009828

6. Pentecost RL, Murray RA, Brindley HH. Fatigue, insufficiency, and pathologic fractures. JAMA 1964;28(187):1001-4. https://doi.org/10.1001/jama.1964.03060260029006 
7. Sakamoto A, Yamamoto T, Tanaka K, Matsuda S, Yoshida T, Iwamoto Y. Insufficiency fracture in the paraacetabulum, with features mimicking those of a malignant bone tumor. Clin Med Case Rep 2008;1:73-6. https://doi.org/10.4137/ccrep.s803

8. Brennan M, O’Shea PM, O'Keeffe ST, Mulkerrin EC. Spontaneous insufficiency fractures. J Nutr Health Aging 2019;23(8):758-60. https://doi.org/10.1007/s12603-019-1234-6

9. Wearing SC, Hennig EM, Byrne NM, Steele JR, Hills AP. Musculoskeletal disorders associated with obesity: a biomechanical perspective. Obes Rev 2006;7(3):239-50. https://doi.org/10.1111/j.1467-789X.2006.00251.X

10. Schachter AK, Roberts CS, Seligson D. Occult bilateral acetabular fractures associated with high-energy trauma and osteoporosis. J Orthop Trauma 2003;17(5):386-9. https://doi.org/10.1097/00005131-200305000-00012

11. Watarai K, Taneda H, Higano M, Hirasawa Y, Oda H. Rapidly destructive arthrosis of the hip joint after insufficiency fracture of the acetabulum. J Orthop Sci 2008;13(6):561-5. https:/doi.org/10.1007/s00776-008-1261-3

12. Angles F, Coscujuela A, Tramunt C, Panisello MG, Portabella F. Complication of an insufficiency fracture of the acetabulum. Hip Int 2008;18(3):236-8. https://doi.org/10.5301/hip.2008.2237

13. Ikemura S, Mawatari T, Matsui G, Iguchi T, Mitsuyasu H. Clinical outcomes in relation to locations of bone marrow edema lesions in patients with a subchondral insufficiency fracture of the hip: a review of fifteen cases. Br J Radiol 2016;89(1066):20150750. https://doi.org/10.1259/bjr.20150750

14. Buttaro M, Della Valle AG, Morandi A, Sabas M, Pietrani M, Piccaluga F. Insufficiency subchondral fracture of the femoral head: report of 4 cases and review of the literature. J Arthroplasty 2003;18(3):377-82. https://doi.org/10.1054/arth.2003.50092

15. Cabarrus MC, Ambekar A, Lu Y, Link TM. MRI and CT of insufficiency fractures of the pelvis and the proximal femur. AJR Am J Roentgenol 2008;191(4):995-1001. https://doi.org/10.2214/AJR.07.3714

16. Guerado E, Cano JR, Cruz E. Occult acetabular fracture in elderly patients. Open Orthop J 2012;6:582-6. https://doi.org/10.2174/1874325001206010582

17. Jo WL, Lee WS, Chae DS, Yang IH, Lee KM, Koo KH. Decreased lumbar lordosis and deficient acetabular coverage are risk factors for subchondral insufficiency fracture. J Korean Med Sci 2016;31(10):1650-5. https://doi.org/10.3346/jkms.2016.31.10.1650

18. Mears DC. Surgical treatment of acetabular fractures in elderly patients with osteoporotic bone. J Am Acad Orthop Surg 1999;7(2):128-41. https://doi.org/10.5435/00124635-199903000-00006

19. Bangil M, Soubrier M, Dubost JJ, Rami S, Carcanagues Y, Ristori JM, et al. Subchondral insufficiency fracture of the femoral head. Rev Rheum Engl Ed 1996;63(11):85-61. PMID: 9010975

20. Lopez Viana S, Machado BB, Mendlovitz PS. MRI of subchondral fractures: a review. Skeletal Radiol 2014;43(11):1515-27. https://doi.org/10.1007/s00256-014-1946-y 